UCRL-JC- 119228

PREPRINT

\title{
SILICA AEROGEL: AN INTRINSICALLY LOW DIELECTRIC CONSTANT MATERIAL
}

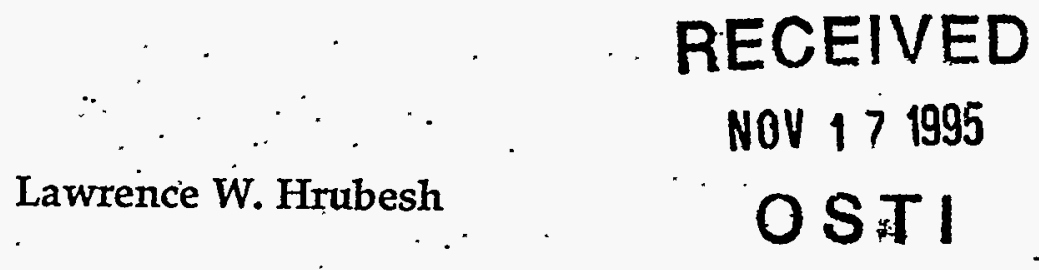

This paper was prepared for submittal to the Materials Research Society 1995 Spring Meeting

San Francisco, CA

April 17-21, 1995

April 1995

This is a preprint of a paper intended for publication in a journal or proceedings. Since changes may be made before publication, this preprint is made available with the understanding that it will not be cited or reproduced without the permission of the author.

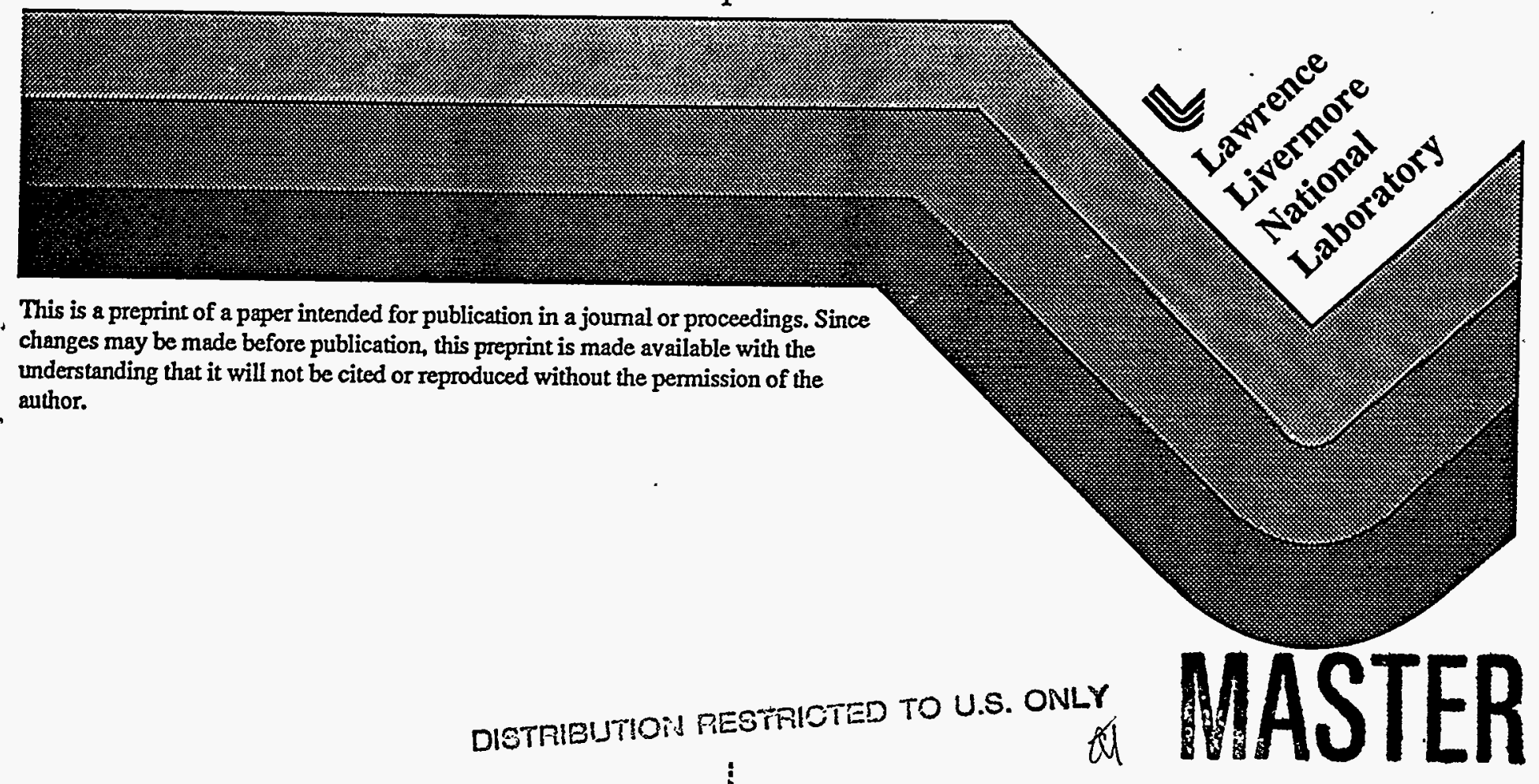




\section{DISCLAIMER}

was prepared as an account of work sponsored by an agency of the United States Government. Neither the United States Government nor the

" $" *$ University of California nor any of their employees, makes any warranty, express or impllied, or assumes any legal liability or responsibility for the accuracy, completeness, or usefulness of any information, apparatus, product, or process is disclosed, or represents that its use would not infringe privately owned rights. Reference herein to any specific commercial product, process, or service by trade name, trademark, manufacturer, or otherwise, does not necessarily constitute or imply its endorsement, recommendation, or favoring by the United States Government or the University of California. The views and opinions of authors expressed herein do not necessarily state or reflect those of the United States Government or the University of California, and shall not be used for advertising or product endorsement purposes. 


\title{
SILICA AEROGEL: AN INTRINSICALLY LOW \\ DIELECTRIC CONSTANT MATERIAL*
}

\author{
LAWRENCE W. HRUBESH \\ Chemistry and Material Science Department \\ Lawrence Livermore National Laboratory \\ Livermore, California 94550
}

\begin{abstract}
Silica aerogels are highly porous solids having unique morphologies in which both the pores and particles have sizes less than the wavelength of visible light. This fine nanostructure modifies the normal transport mechanisms within aerogels and endows them with a variety of exceptional physical properties. For example, aerogels have the lowest measured thermal conductivity and dielectric constant for any solid material. The intrinsically low dielectric properties of silica aerogels are the direct result of the extremely high achievable porosities, which are controllable over a range from $75 \%$ to more than $99.8 \%$, and which result in measured dielectric constants from 2.0 to less than 1.01. This paper discusses the synthesis of silica aerogels, processing them as thin films, and characterizing their dielectric properties. Existing data and other physical characteristics of bulk aerogels (e.g., thermal stablity, thermal expansion, moisture adsorption, modulus, dielectric strength, etc.), which are useful for evaluating them as potential dielectrics for microelectronics, are also given.
\end{abstract}

* Work performed under the auspices of the U.S. Department of Energy by the Lawrence Livermore National Laboratory under Contract No. W-7405-ENG-48. 


\section{INTRODUCTION}

New dielectric substrate materials which exhibit low dielectric permittivity (low-k) compared to presently used dielectrics, will be critical in the development path towards faster and power efficient microelectronics. For example, low-k materials used in interconnects will enable lower power usage through reduction of both nodal and interconnect line capacitances. Since reduced capacitance proportionally lowers dynamic power consumption in circuits, the development of low-k dielectric materials has been identified as a critical component of the multilevel circuit technology roadmap. For these reasons, the next generation of dielectric substrate materials should have low dielectric constants $(<2.5)$ and future materials should have ultra-low dielectric constants $(<2.0)$. Currently, there are ongoing efforts in both industry and government to develop low-k materials. Some of the work involves developing new organic polymers but temperature limitations of organics are also driving the development of more thermally stable inorganic materials. For both types, significant lowering of the permittivity can be obtained with increased porosity in which a greater fraction of the bulk volume contains air. Porosity in solids can be achieved in a variety of ways but the preferred method would directly form materials having both uniform porosity and small spatial entities (e.g., $<100 \mathrm{~nm}$ ). One such method is sol-gel chemistry, which is conventionally used to make homogeneous, low porosity materials in either bulk or film form.

Aerogels are a special class of sol-gel derived materials which are dried in a way that achieves very high porosity even though the pores and particles are smaller than wavelengths of visible light. The pores are open and interconnected in aerogels. This unique nanostucture is responsible for their exceptional dielectric properties as well as for other complementary properties useful for microelectronic applications. Aerogels can be made from many different chemical compositions, including both organic and inorganic varieties, but the most common is silica aerogel. This paper will discuss the intrinsic dielectric properties of silica aerogels, briefly describe how they are made, fabricated as thin films, metallized, patterned, and characterized. Properties of silica aerogels will be compared with those of other porous dielectric materials. 


\section{INTRINSIC DIELECTRIC PROPERTIES OF SILICA AEROGELS}

Silica aerogels have intrinsically low dielectric permittivity for the following reasons:

- they are intrinsically porous because of their formation by sol-gel chemistry

- they consist of two interconnecting phases, both of which are excellent dielectrics

- the major phase is air which dominates the bulk dielectric properties

- the $\mathrm{SiO}_{2}$ phase is pure, free from ionic impurities

- they are homogeneous on a nanometer scale and isotropic

- their unique morphology limits charge mobility within them

Silica aerogels have intrinsic porosity; that is, their structure is formed from molecular sized clusters which themselves have microporosity, and which are connected in such a way that a three dimensional structure forms without having pores larger than a few cluster diameters. Silica aerogel may thus be classified as a solid even though the distributed solid mass occupies only a fraction of its total volume. Figure 1 shows a simplified structural model for silica aerogel in which two levels. of porosity are identified; microporosity $(<2 \mathrm{~nm})$ within primary particles, and mesopores $(<150 \mathrm{~nm})$ between particles. The gel is composed of two continuous phases, the interconnected solid and the pore-filling air. Since each phase is continuous through a random structure, the bulk aerogel can be represented in an equivalent circuit as either series or parallel connected. Each phase can be considered as a pure dielectric, but with a common interface. 


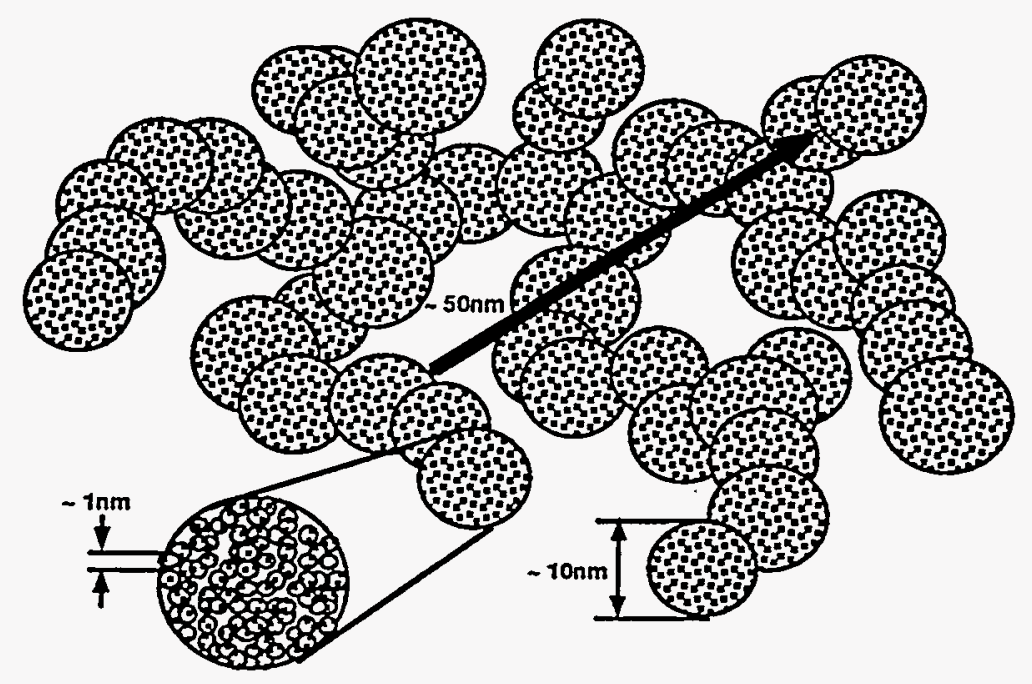

Figure 1. Simplified structural model for silica aerogel showing two levels of porosity

All of the existing permittivity data (given below) for silica aerogels having $>75 \%$ porosity, show that the dielectric constants are nearly proportional to the bulk density (or porosity). This linear behavior is predicted on the basis of molecular polarizability if the pore-filling gas phase is the dominant phase. For example, from the Clausius-Mosotti relation [1] the dielectric constant is given by, $k^{\prime}-1=(K \alpha) p$, where $\alpha$ is the molecular polarizability, $\rho$ is the density, and K is a constant obtained from molecular parameters. Linear behavior is also predicted from a simple parallel mixing rule model where the two phases are considered to have parallel capacitances in the field direction. The following assumptions are applied; the two constituent phases have dielectric properties as though they are pure single phases, interfacial effects are neglected (although this assumption is weak for aerogels because of the significant surface area available for sorption of gases, particularly water), and both phases are perfect insulators. Again, if the gas phase is dominant, the parallel mixing model gives, $\kappa^{\prime}-1=\left[\left(\kappa s^{\prime}-1\right) / \rho s\right] \rho$, where $\kappa s^{\prime}$ and $\rho$ s are the dielectric constant and density of the solid phase, respectively. For silica, this relation is $\kappa^{\prime}-$ $1=1.42 p$; our data for dried silica aerogel, measured at microwave frequencies [2] gives $\kappa^{\prime}-1 \approx 1.48 p$, in good agreement with this relation. It can also be shown [1] that for gas molecules, the loss factor, $\kappa^{\prime \prime}$, is proportional to the number of molecules per unit volume and therefore it is also proportional to the density, $\rho$. So the loss 
tangent is, $\tan \delta=\kappa^{\prime \prime} / \kappa^{\prime}=c(\rho / 1+\rho)$, where $c$ is a constant. Then, for very small $\rho$, the loss tangent is also proportional to the density, $\rho$.

Thus, it appears that the high volume ratio of gas-filled voids in silica aerogels gives them the intrinsically low dielectric properties associated with the gas phase. This still seems to be the case even when water adsorbs on the large internal surface area of the aerogel and affects the dielectric properties, because it does not appear to significantly modify the linearity with density.

\section{SILICA AEROGEL SYNTHESIS AND PROCESSING}

\section{Bulk Silica Aerogels}

The sol-gel chemistry used to prepare silica aerogels has been well-studied and documented [3]. Briefly, the preferred process involves the hydrolysis and condensation of silicon alkoxides (e.g., tetramethoxysilane, TMOS, or tetraethoxysilane, TEOS) in a single-step mixing procedure where the alkoxide, water, a small amount of base catalyst, and additional amounts of alcohol for density control are mixed together. For example, a typical molar ratio for a density of $0.2 \mathrm{~g} / \mathrm{cc}$ is, 1 TMOS: $4 \mathrm{H}_{2} \mathrm{O}: 10^{-5} \mathrm{NH}_{4} \mathrm{OH}: 4 \mathrm{MeOH}$. The alcohol is added as a diluent to the reaction, and high porosity gels normally contain more than $75 \%$ solvent. The mixture is poured into a glass mold, sealed, and allowed to gel overnite at room temperature.

The next step to achieve a silica aerogel is to dry the alcohol-filled gel without collapsing its tenuous structure. Such drying is normally accomplished by supercritical extraction (SCE) of the solvent, but recent reports describe successful silica aerogels by evaporative drying in air $[4,5]$. In the SCE process, the gel is placed in a pressure vessel and heated under pressure until the alcohol becomes a supercritical fluid (i.e., $P_{c} \approx 8 \mathrm{MPa}$ and $T_{c} \approx 250^{\circ} \mathrm{C}$ ). The supercritical fluid is then

slowly removed from the gel and replaced with air, while the vessel is cooling back to room temperature. The typical time required to complete this drying process is about $20 \mathrm{hrs}$ for $500 \mathrm{~cm}^{3}$ silica aerogel pieces. An alternative lower temperature SCE process (i.e., $\mathrm{T}_{\mathrm{c}} \approx 32^{\circ} \mathrm{C}$ and $\mathrm{P}_{\mathrm{c}} \approx 7.4 \mathrm{MPa}$ ) can be done with $\mathrm{CO}_{2}$ as the gel fluid after it is exchanged for the alcohol. The silica aerogels from high temperature SCE process are hydrophobic (i.e., not wetted by water) whereas; the $\mathrm{CO}_{2}$ process yields hydrophilic aerogels. 


\section{Thin Film Silica Aerogels}

The ability to form thin films of silica aerogels on silicon substrates is necessary for its practical use as a low dielectric constant material in microelectronics. Thin films of silica aerogel having thicknesses of less than $1 \mu \mathrm{m}$ to several $\mu \mathrm{m}$, have been successfully made on silicon substrates at LLNL by using conventional spinning and dipping methods performed in enclosures which were maintained with a saturated alcohol atmosphere [6]. The controlled environment prevents rapid evaporative drying which would densify the gel after it forms on the substrate. The gel-coated substrates are dried by SCE to produce high porosity silica aerogel films. Separate authors have recently reported simpler processes for airdrying silica aerogel films [4,5]. These new processes represent a significant breakthrough by eliminating the need for SCE and because they enable use of conventional spin-on-glass processing for thin aerogel films.

\section{Metallization and Patterning}

The surfaces of as-prepared silica aerogel are sufficiently smooth to allow direct metallization by vapor or sputter deposition. LLNL has demonstrated low resolution metal patterns produced by vapor deposition through masks onto silica aerogels [7]. We have also sputtered thin $(<0.5$ micron) metal layers onto the silica aerogel surfaces and electroplated thicker ( $>1.0$ micron) layers. Photoresist. patterning techniques were also successfully applied but only after the aerogel surfaces were coated with approximately $100 \mathrm{~nm}$ of parylene, to seal the pores of the aerogel. Qualitative tests show that the aerogels can be sealed from subsequent semiconductor process liquids so that conventional photoresist techniques can be used to patiern the aerogels.

\section{CHARACTERIZATION OF SILICA AEROGEL DIELECTRICS}

To date, the dielectric properties of aerogels have not been studied as extensively as other properties but some data are available for silica aerogels. Dielectric properties, including the dielectric constant, loss tangent, dielectric conductivity, volume resistivity, and dielectric strength, have been reported for bulk silica aerogels $[2,8,9]$. Characterizing silica aerogel films is difficult, generally 
requiring optical techniques which are less effective for silica aerogels than for more dense silica coatings. There are only a few reports of dielectric constant measurements made for thin silica aerogel films $[4,5,6]$.

\section{Dielectric Permittivity Measurements}

Permittivity measurements are reported for a variety of sol-gel derived porous silica materials $[10,11]$, including silica aerogels $[2,4,5,8,9]$. Collectively, these measurements cover a wide frequency range from $10 \mathrm{~Hz}$ to $40 \mathrm{GHz}$ and confirm the. low permittivity values expected for such highly porous materials.

For bulk silica aerogels, measurements of the dielectric constant and loss factor were reported by daSilva, et al. [8] for frequencies between .50 and $10^{5} \mathrm{~Hz}$, and for temperatures of $1.6 \mathrm{~K}$ to $300 \mathrm{~K}$. Brüesch, et al. [9] measured bulk silica aerogels at $10^{3} \mathrm{~Hz}$. We have measured permittivities for silica aerogels, at microwave frequencies (i.e., 2 to $40 \mathrm{GHz}$ ) and at $298 \mathrm{~K}$ [2].

For thin silica aerogel films, dielectric constant values have been inferred from optical refractive index measurements. At LLNL, we measure the optical refractive index, $n$, of silica aerogel films using ellipsometry, then calculate the density, porosity, and dielectric constants using empirical relations for each, which have been determined to hold for bulk silica aerogels. For example, the density is found from the empirical relation, $\rho=(n-1) / .209$, reported for silica aerogels [12]. The porosity is determined from, $\Pi=1-\rho / \rho$, then using $\rho s=2.2 \mathrm{~g} / \mathrm{cc}$ for silica, and the above relation, $\Pi=[(1.46-n) / 0.46] \times 100$ is the percent porosity. Similarly, the dielectric constants for silica aerogel films are determined by using, $\kappa^{\prime}=1+1.6 p[2]$ and the above relation, so that $\kappa^{\prime}=1+7.7(n-1)$.

To summarize, all reported measurements for dielectric constant give values less than 2.0 for silica aerogel porosities $>75 \%$. In that range, all measurements are consistent with the parallel (or linear) model of dielectric constant vs density. Loss tangent measurements also approximately fit a linear model; the range is from $5 \times 10^{-4}$ to $8 \times 10^{-2}$ depending on the density. We have published empirical relations for as-prepared silica aerogels, $\kappa^{\prime}=1.6 \rho$ and $\tan \delta=1.72 \times 10^{-4} \rho$, and for dry silica aerogels, $\kappa^{\prime}=1.48 \rho, \tan \delta=4.3 \times 10^{-5} \rho$ ( $\rho$ has units of $\mathrm{kg} / \mathrm{m}^{3}$ ). We note that, at microwave frequencies, the adsorbed water inside the aerogel accounts for about $7 \%$ of the dielectric constant value and about $70 \%$ of the loss tangent value. 


\section{Dielectric Conductivity, Volume Resistivity and Dielectric Strength}

The dielectric conductivity $(\sigma)$ of aerogels is obtained from our microwave measurements by using the relation [1], $\sigma=5.5 \times 10^{-13} \kappa^{\prime} \tan \delta \mathrm{f}(\mathrm{ohm}-\mathrm{cm})$, where $\kappa^{\prime}$ and $\tan \delta$ are the dielectric constant and the loss tangent, respectively, and $f$ is the frequency. For dry silica aerogel with a density of $100 \mathrm{~kg} / \mathrm{m}^{3}$, the dielectric conductivity ranges from $1.1 \times 10^{-4}$ to $8.1 \times 10^{-6} \Omega^{-1} \mathrm{~cm}^{-1}$ in the frequency range from 3 to $40 \mathrm{GHz}$. The typical volume resistivity for dry silica aerogels (i.e., $2 \pi \mathrm{f} / \mathrm{s}$ ) with the same density, is $4.1 \times 10^{15} \Omega \mathrm{cm}$. These values of volume resistivity for aerogels are higher than for most porous materials and are comparable with the best of the polymer insulating materials (e.g., polytetrafluoroethylene and polyethylene) [13]. Aerogels should therefore be expected to exhibit very good dielectric strength against high voltage breakdown.

Aerogels exhibit higher dielectric strength than expected for porous materials. This is probably attributable to the pore sizes in aerogels being of the same order as the mean-free-path for electron collisions. Electrons in aerogel pores tend to collide with the solid before gaining sufficient kinetic. energy to ionize upon impact. Brüesch, et.al. [9] measured a dielectric strength of $500 \mathrm{kV} / \mathrm{cm}$, for a bulk silica aerogel $\left(\rho=180 \mathrm{~kg} / \mathrm{m}^{3}\right)$, at $50 \mathrm{~Hz}$, in a $\mathrm{SF}_{6}$ gas. We have measured an average dielectric strength of $128 \mathrm{kV} / \mathrm{cm}$ for air-filled silica aerogels ( $\rho=120$ to $200 \mathrm{~kg} / \mathrm{m}^{3}$ ), at $60 \mathrm{~Hz}$. These values are higher than for most ceramics but less than for pure polymers. Silica aerogels should be effective lightweight high voltage insulators, especially in vacuum applications.

\section{Other Properties and Property Comparison}

Three other properties of aerogels are important for applications of dielectrics in electronics; 1) sufficiently high thermal conductivity for heat dissipation, 2) a thermal expansion which closely matches the substrate to reduce stress-induced cracking, and 3) sufficient shear and compressive strength to support multiple layers. While aerogels are notable for their excellent thermal insulation property, the higher density silica aerogels actually have comparable thermal conductivities with some of the polymer films used for low dielectrics. The coefficient of thermal expansion for silica aerogel is $\sim 3 \times 10^{-6} \mathrm{~cm} / \mathrm{cmK}$ (for $\mathrm{T}=275-323 \mathrm{~K}$ ). This value is near that of fused silica, so that silica aerogel should be thermally compatible with the 
coatings used in electronics packaging. Lastly, the shear strength has not been adequately measured for silica aerogels, but they are known to be weak in tension. However, their compressive strength increases sharply with density, and strength should only be an issue for the lowest density silica aerogels (i.e., $<200 \mathrm{~kg} / \mathrm{m}^{3}$ ).

To date, with the exception of dielectric properties, bulk silica aerogels have been well characterized. Comprehensive results can be found in a recent review article [14]. As for their dielectric properties, silica aerogels clearly are exceptional when compared with other porous dielectric materials. Such comparison is made in graphic form in figure 2. Maximum and minimum values for each category show that silica aerogels match or exceed other porous materials for properties which are important in microelectronics. However, other important issues await the results of further investigations.

\section{CONCLUSIONS}

It has been shown that silica aerogels are intrinsically low dielectric constant materials and existing data on bulk aerogels also confirm that they meet or exceed some of the other property requirements for use in integrated electronics such as: thermal stability, moisture absorption, low thermal expansion, compatibility with conductors, no UV degradation. However, further work is needed to determine if silica aerogels can be adapted or modified to meet the processing requirements for integrated electronics and if integrating aerogels into microelectronics can be cost effective. 


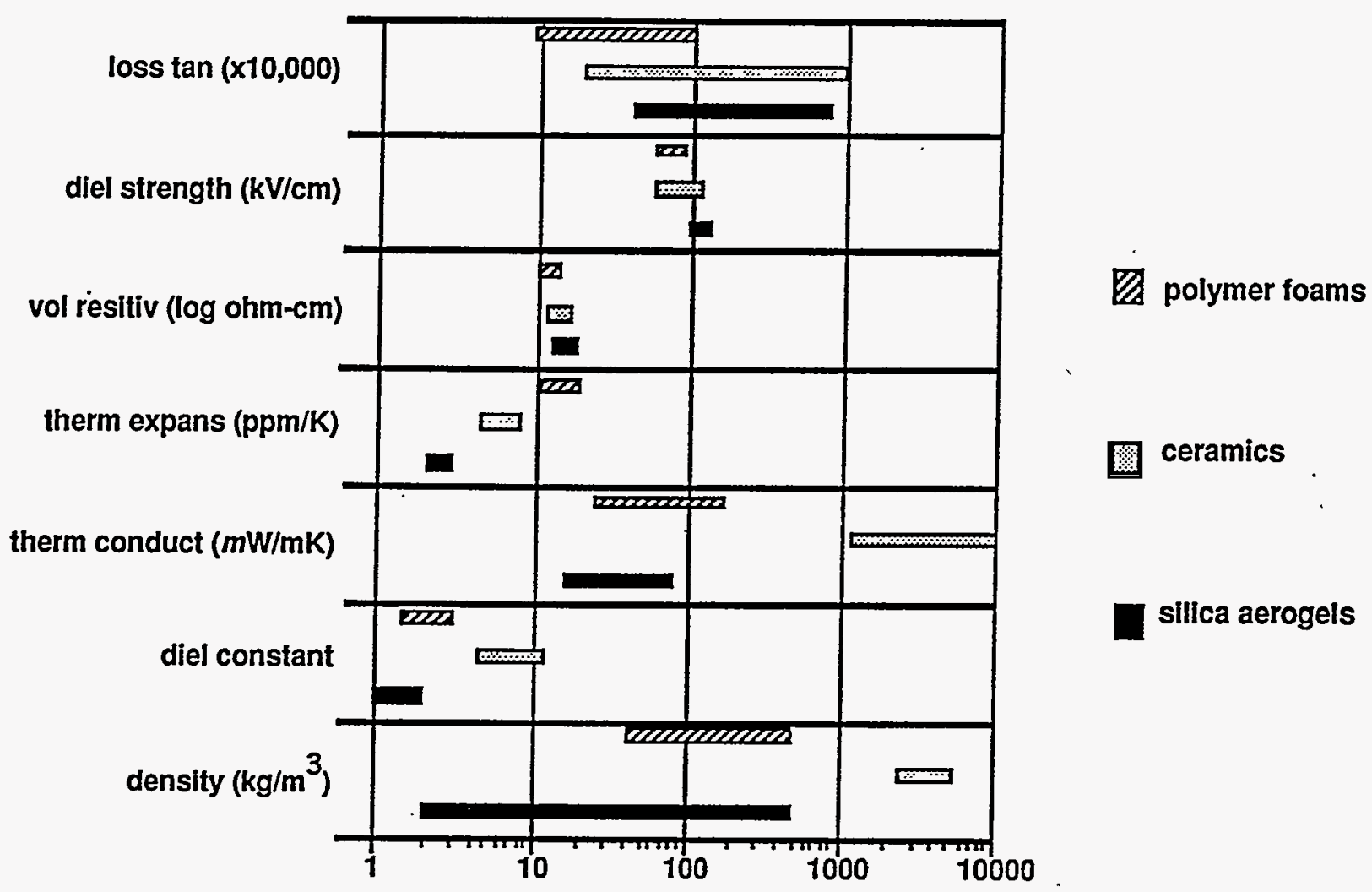

Figure 2. Comparison of properties for three types of porous dielectric materials

\section{References:}

1. A.R. von Hippel, Dielectric and Waves, (J.Wiley and Sons; New York, 1954), p. 28.

2. L.W. Hrubesh, L.E. Keene, and V.R. Latorre, J. Mater. Res. 8, 1736 (1993).

3. C.J. Brinker and G.W. Scherer, Sol-Gel Science (Academic Press, N.Y., 1990) p. 97.

4. S.S. Prakash, C.J. Brinker, A.J. Hurd and S.M. Rao, in Advances in Porous Materials, edited by S. Komarneni, D.M. Smith, and J.S. Beck, (Mat. Res. Soc. Symp. Proc., Vol. 371, Mat. Res. Soc., Pittsburgh, 1995) pp. 205-210.

5. D.M. Smith, J. Anderson, C.C. Cho, and B.E. Gnade, in Advances in Porous Materials, edited by S. Komarneni, D.M. Smith, and J.S. Beck, (Mat. Res. Soc. Symp. Proc., Vol. 371, Mat. Res. Soc., Pittsburgh, 1995) pp. 261-266. 
6. L.W. Hrubesh and J.F. Poco, in Advances in Porous Materials, edited by S. Komarneni, D.M. Smith, and J.S. Beck, (Mat. Res. Soc. Symp. Proc., Vol. 371, Mat. Res. Soc., Pittsburgh, 1995) pp.195-204.

7. L.W. Hrubesh and J.F. Poco, J. Non-Cryst. Solids, (to be published).

8. A. daSilva, D.I. dosSantos, and M.A. Aegerter, J. Non-Cryst. Solids 96, 11591166 (1987).

9. P. Brüesch, F. Stucki, Th. Baumann, P. Kluge-Weiss, B. Brühl, L. Niemeyer, R. Strümpler, B. Zeigler and M. Mielke, J. Appl Phys. A, 57, 329-337 (1993).

10. W.A. Yarbrough, T.R. Guruaja, and L.E. Cross, Am. Ceram. Soc. Bull. 66, 692 (1987).

11. W. Cao, R. Gerhardt, and J.B. Wachtman, in Advances in Ceramics, edited by M.F.Yan, K. Niwa, H. Obryan, Jr. and W.S. Young (Amer. Ceram. Soc., Ohio, 1989), pp. 409-418.

12. S. Henning and L. Svensson, Phys. Scripta 23, 697 (1981).

13. D.W. Van Krevelen, Properties of Polymers, (Elsevier, New York, 1990), pp. 330-333. 14. J. Fricke and A. Emmerling, in Chemistry, Spectroscopy, and Applications of Sol-Gel Glasses, edited by R. Reisfeld and C.K. Jorgensen, (Springer Series on Structure and Bonding, Vol. 77, Springer-Verlag, Heidelberg, Germany, 1991) p.37. 

Technical Information Department $\cdot$ Lawrence Livermore National Laboratory University of California - Livermore, California 94551

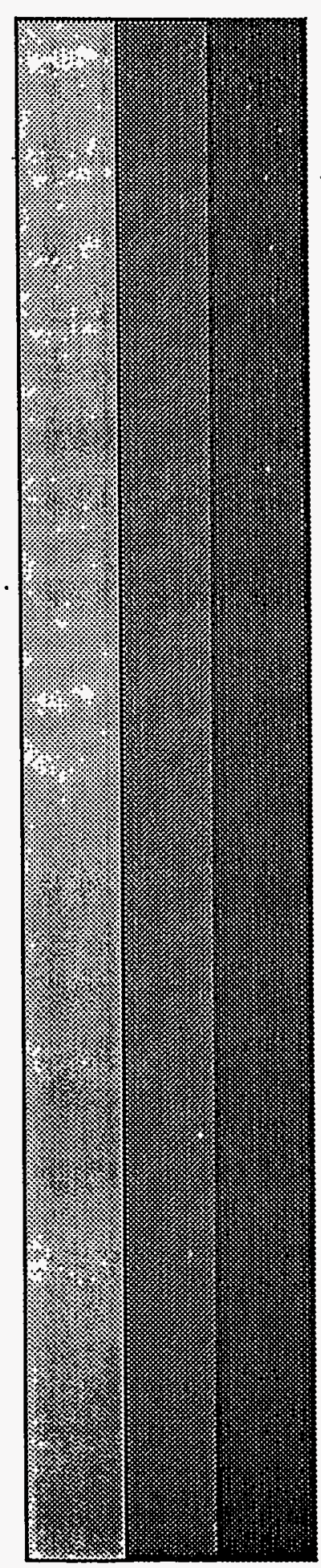

